\title{
Questions à la Commission européenne
}

Entretien avec Madame Michèle Sellier, expert national détaché à la DG XXII, chargée du suivi du Livre blanc

Martine Faure et Michel Massacret

\section{(2) OpenEdition}

1 Journals

Édition électronique

URL : http://journals.openedition.org/ries/3056

DOI : $10.4000 /$ ries.3056

ISSN : 2261-4265

Éditeur

Centre international d'études pédagogiques

Édition imprimée

Date de publication : 1 décembre 1997

Pagination : 119-122

ISSN : 1254-4590

\section{Référence électronique}

Martine Faure et Michel Massacret, "Questions à la Commission européenne », Revue internationale d'éducation de Sèvres [En ligne], 16 | Décembre 1997, mis en ligne le 10 juin 2013, consulté le 30 avril 2019. URL : http://journals.openedition.org/ries/3056 ; DOI : 10.4000/ries.3056

Ce document a été généré automatiquement le 30 avril 2019.

(c) Tous droits réservés 


\section{Questions à la Commission européenne}

Entretien avec Madame Michèle Sellier, expert national détaché à la DG XXII, chargée du suivi du Livre blanc

\section{Martine Faure et Michel Massacret}

\section{NOTE DE L'ÉDITEUR}

Propos recueillis par Martine Faure et Michel Massacret

Le Livre blanc Enseigner et apprendre - Vers la société cognitive a suscité un large débat que vous avez suivi pour la DG XXII.

Compte tenu de l'importance des implications philosophiques et sociales de la réflexion engagée par le Livre blanc, les mots pour le dire - et en plusieurs langues - ont été euxmêmes matière à débat. L'expression « société cognitive » n'a pas eu que des adeptes, la formule ayant paru à certains trop étroite. Un terme a-t-il pu obtenir un consensus et élargir le champ?

En effet, le terme " cognitif » a suscité des réserves et la traduction retenue en anglais "Learning Society" renvoie d'ailleurs à une autre notion, celle de la "société apprenante ». Dans le premier bilan réalisé par la Commission, les valeurs éthiques fondant cette démarche ont été soulignées. Le Parlement européen a insisté, pour sa part, sur ses aspects sociaux, moraux et spirituels.

Maintenant, c'est le terme « Europe de la connaissance $»^{1}$ qui a été adopté et qui figure dans le traité d'Amsterdam. Son préambule stipule notamment que le traité de l'Union « s'est donné comme objectif de développer l'éducation et la formation tout au long de la vie, c'est-à-dire sa volonté de promouvoir le développement du niveau de connaissance le plus élevé pour son peuple par un plus large accès à l'éducation et à la mise à jour permanente des connaissances ».

Pourquoi le Livre blanc aujourd'hui ? La question de la formation tout au long de la vie, outre la nécessité pour l'Europe du XXe siècle de se donner un potentiel humain hautement qualifié, ne cache-t-elle pas la question d'une « employabilité » réduite, que ce soit pour les 
jeunes ou les cadres enfin de carrière ? Dans ce cas, quelles formes de solidarité proposer à nos concitoyens et quels moyens envisager?

La question du chômage préoccupe au plus haut degré la Communauté européenne. Je vous renvoie pour la question sur ce thème aux résultats du sommet du Luxembourg ${ }^{2}$ consacré à l'emploi, qui a insisté sur la nécessité de développer « l'aptitude de l'homme à l'emploi ", expression préférée à celle d' « employabilité ». Lors de ce sommet, le développement de la formation professionnelle tout au long de la vie s'est trouvé au cœur des débats. Le rapport de la Commission intitulé L'accès à la formation continue dans l'Union européenne, fondé sur une synthèse des rapports nationaux, en souligne les enjeux.

Quelles évolutions constate-t-on dans les dispositifs actuels de formation? La formation permanente et continue - après toute une période fortement axée sur l'emploi - revient-elle à des missions de développement personnel ou à des préoccupations de (re)socialisation des individus? Quelle part notamment prennent les nouveaux dispositifs en cours d'expérimentation, notamment ceux développant la capacité d'apprendre à apprendre, les formes variées d'individualisation et la formation ouverte et à distance?

Il s'agit bien en effet de favoriser pour tous l'accès à la connaissance. Ainsi cherche-t-on à encourager toutes les nouvelles voies que vous citez, afin de prendre en compte la diversité des situations et de responsabiliser davantage les individus dans le déroulement de leurs apprentissages.

Dans le plan d'action «Apprendre dans la société de l'information », qui suit le Livre blanc, il est question de donner "plus de transparence » aux compétences et aux qualifications requises. La formule est également utilisée dans le Livre vert consacré aux « obstacles à la mobilité transnationale ». Où en est-on dans le développement du parcours européen de formation pour l'apprenti? Quels sont les moyens envisagés pour favorise davantage la mobilité ?

Des progrès remarquables en matière d'équivalence des diplômes et de mobilité sont déjà acquis: selon les chiffres publiés récemment, la plupart des universités sont maintenant reliées entre elles grâce à Erasmus. Le système ECTS ${ }^{3}$ permet une reconnaissance mutuelle des périodes d'études effectuées à l'étranger. Des progrès ont été réalisés en matière d'équivalence de diplômes. La situation est moins avancée pour la formation professionnelle. Le Livre vert, quant à lui, s'attache à la mobilité et à la levée des obstacles techniques de nature fiscale, en particulier. Enfin, pour ce qui est du parcours européen de l'apprenti, le projet de décision est désormais adopté.

Concernant l'accréditation des compétences, s'est-on mis d'accord sur une définition européenne de la compétence? Comment la «carte personnelle des compétences » estelle admise aujourd'hui par les universitaires? Enfin, comment l'automaticité des procédures d'évaluation est-elle accueillie par les milieux professionnels?

Ces questions ont déjà suscité de nombreuses réactions. Il n'y a pas de définition européenne de la compétence; on dispose, cependant, pour certains métiers, de référentiels. S'agissant du débat autour des compétences et des qualifications, il est difficile de se mettre d'accord sur une terminologie commune. La Commission s'est principalement attachée à la reconnaissance des qualifications socialement reconnues : le Livre blanc a proposé une carte personnelle des compétences qui valorise les modes d'accréditation, sans remettre en cause les diplômes. Des expérimentations sont en cours dans un esprit très pragmatique. Ainsi, des universitaires réfléchissent à la conception et à la réalisation de logiciels d'autoévaluation. Cette démarche tout à fait novatrice, devra être débattue par l'ensemble des partenaires sociaux. 
Les écoles de la deuxième chance suscitent beaucoup de commentaires. Faut-il en déduire une remise en cause de la capacité des écoles ordinaires à accueillir tous les enfants? $\mathrm{Ne}$ risque-t-on pas de stigmatiser l'exclusion en créant des modes d'assistanat permanent et des voies d'accès à l'emploi à deux vitesses ?

Le problème est d'importance. Madame Cresson considère qu'il faut faire face aux réalités. D'après les chiffres de l'OCDE, $20 \%$ de la population n'aurait pas les connaissances de base nécessaires à une poursuite de formation à l'âge adulte. Les écoles de la deuxième chance sont organisées actuellement de manière à expérimenter des moyens de lutte contre l'exclusion. Tous les États membres ont demandé à créer de telles écoles ou sont en cours de discussion avec la Commission sur ce sujet.

La recommandation d'apprendre deux langues communautaires en plus de la langue maternelle ne risque-t-elle pas de créer un repli identitaire européen, alors que I'Union européenne se positionne de plus en plus comme un acteur international ? Que faire de langues telles que le russe, l'arabe, le chinois, parlées par de larges communautés? Comment aborder les cultures et les marchés non-européens ?

La Commission a été interrogée sur cette question par le Parlement européen. La réponse qu'il convient d'apporter est la suivante : La Commission considère qu'elle a vocation à encourager l'apprentissage des onze langues européennes officielles, dans le cadre de l'Europe des Quinze. La Commission considère, en effet, qu'elle doit œuvrer pour la reconnaissance d'un même statut des langues communautaires et qu'elle doit porter une attention spécifique à la pratique des langues modimes ${ }^{4}$. Chaque État est bien entendu encouragé vivement à développer l'apprentissage et la pratique d'autres langues que celles de l'Union, dans le cadre de sa propre politique linguistique et dans le respect du principe de subsidiarité.

Comment la Commission entend-elle étendre aux Pays d'Europe centrale et orientale (PECO) les acquis des programmes actuels dont les opérateurs européens soulignent déjà l'insuffisance des moyens?

Les programmes éducatifs communautaires s'ouvrent progressivement aux pays en voie d'adhésion. Effectivement, même sans prendre en compte les demandes des PECO, les moyens semblent déjà insuffisants au regard des actions à entreprendre. En ce qui concerne l'augmentation souhaitée du budget du programe Socrates, la question est actuellement débattue entre le Conseil Éducation ${ }^{5}$ et le Parlement européen.

Souhaitez-vous souligner d'autres commentaires ou d'autres évolutions à propos du Livre blanc?

Au moment où la Commission réfléchit à l'avenir des programmes européens pour l'an 2000, différentes publications de la Commission dont la communication Pour une Europe de la connaissance et le document de travail Les acquis des programmes, sont disponibles sur Internet. J'encourage toutes les personnes intéressées par ces questions majeures pour l'avenir de l'Europe, à prendre connaissance de ces textes ou à les consulter. 


\section{NOTES}

1. (Com (97) 256 final).

2. Novembre 1997.

3. European Credit Transfer System.

4. Moins diffusées et moins enseignées.

5. Conseil réunissant les ministres chargés des questions d'éducation de tous les États membres.

INDEX

Index géographique : Europe

Mots-clés : formation tout au long de la vie, politique européenne, politique éducative

\section{AUTEURS}

\section{MARTINE FAURE}

Chargée de mission pour le développement international, rectorat-DAFCO, académie de Lille, France

\section{MICHEL MASSACRET}

Responsable du département de didactique du français langue étrangère, CIEP, France. 